\title{
Replicae of Antibodies - robust mass-sensitive Sensors for Allergens and other Bioanalytes
}

\author{
F.L. Dickert, R. Schirhagl, P.A. Lieberzeit, T.F. Hussain, and M. Cichna-Markl \\ Department of Analytical Chemistry and Food Chemistry, University of Vienna \\ Währingerstrasse 38, A-1090 Wien, Austria
}

\section{Introduction:}

Food allergies represent a severe health problem in industrialized countries. They affect up to 2 $\%$ of the adult population and $8 \%$ of children. ${ }^{1}$ Sesame, which affiliates to the family of pedaliaceae, contains one of the most important food allergens. Hulled seeds are dried or roasted and added to various kinds of food. Products as crackers, bread, fast food or sweets contain sesame. The rising consumption of sesame leads to contamination of other comestibles as well. Sesame seeds comprise of $50 \%$ sesame oil and $25 \%$ sesame protein, which contains at least 10 allergenic proteins, and other compounds. ${ }^{2,3}$ The protein contains $65-70 \% \alpha$-globulin and $30-35 \% \beta$-globulin ${ }^{4}$ and has an isoelectrical point of 4.9 and consists of 12 subunits with masses of $80000-85000$ Da resulting in a mass of 250000 $\mathrm{Da}$ for the whole protein. The increasing importance of sesame allergy requires accurate, safe, and costeffective diagnostic techniques.

The common techniques for the detection of sesame are polymerase chain reactions (PCR) of encoding DNA or enzyme linked immuno sorbent assay (ELISA). Natural antibodies as used for ELISA are well established molecular recognition systems for hazardous chemicals, proteins or viruses. They are known for their selectivity and high affinities to their antigens, which is due to the optimal fit between the binding site and the antigen structure.

However, the generation of immunoglobulin coatings is accompanied by appreciable time consuming efforts. Additionally, for sensor application natural immunoglobulins require a special treatment to break the bond between antigen and antibodies to make the sensor response reversible. Furthermore, natural materials tend to degrade since proteins include oxidizable groups leading to denaturising.

These problems can be solved when the recognition capabilities of natural antibodies are transferred to organic polymers, which are more stable than biological materials. In this way immunological responses are cast in polymers which are established in a long evolution period. Such materials, which are used as stationary phases in chromatography or catalysts, can also be used as sensor materials. ${ }^{5}$ They allow mass sensitive and label free detection of bioanalytes as proteins ${ }^{6}$, viruses ${ }^{7}$ and cells ${ }^{8}$ as coatings on quartz crystal microbalances or surface acoustic wave (SAW) resonators. Therefore, beads ${ }^{9}$ in the size of $20-600 \mathrm{~nm}$ are pre-polymerised and precipitated in presence of natural immunoglobulins. The nanoparticles are washed to remove the antibody leaving holes with imprints of the antibody behind. Finally, these particles are adhered on a glass plate, dried and pressed into another polymer placed on the surface of a QMB. After removing the stamp polymer with the positive structure of the antibody, the replica is left.

\section{Measurements of sesame with natural antibodies}

For comparison to artificial antibodies, natural ones were tested for the measurement of sesame in food samples. Immunoglobulin-Y, extracted from eggs layed by immunised hens, was chosen for this application because identical antibodies in high quality are produced, which can be extracted rather straight forwardly.

The IgY is analogue to immunoglobulin-G in human blood. Human blood contains 5 classes of immunoglobulins: A, D, G, M and E which differ in functions and types of heavy chains. Immunoglobulin-A is localised in liquors like lacrimal fluid. Immunoglobulin-E plays an important role in allergic reactions. The M-type, which forms pentamers, is responsible for early reactions with new antigens and is localised on B-cells (type of white blood cells). The function of the D-Type is not understood. The immunoglobulin$G$ is the most prominent antibody in the blood plasma and the only one which is transferred through the placenta barrier for the protection of the foetus. Immunglobulins- $G$ and their analogues in eggs, which are used in this work, consist of 4 chains. The tetramer of immunoglobulin- $G$ consists of 2 heavy chains (50000-70000 Da) and 2 light chains (25000 Da) forming an upsilon. The protein is shaped like a lentil with a diameter of about $15 \mathrm{~nm}$ and a height of $3 \mathrm{~nm}$ and has a molecular mass of about $150000 \mathrm{Da}$. 
Parts of one light and one heavy string, connected by disulfide bridges, form the fab-fragment which is the

Immobilisation of natural antibody and measurement of sesame

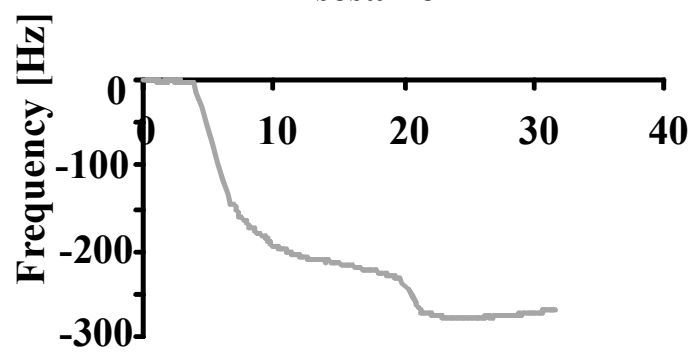

Time [min]

Fig. 1: Frequency change of a $10 \mathrm{MHz}$ QCM. The first step shows the adsorption of antibody the second the binding of sesame.

binding site. Consequently, there are 2 binding sites per immunoglobulin. The fab-fragment is variable and specific for its antigen whereas the rest of the chains are equal for different types of immunoglobulins. Commercial ELISA systems show high affinities resulting in low detection limits in ppm range.

Natural immunoglobulins- $Y$ were immobilised on a gold surface by adhesion of sulphur to the gold surface. Due to the high affinity of sulphur to gold, the immobilised antibodies are tightly bound and not washed out in water or aqueous solutions. Figure 1 shows the QCM-frequency response with sesame of immunoglobulin-Y linked to the gold electrodes. The first step shows the adhesion of antibody the second represents the binding of sesame. To recover the sensor it is necessary to wash the sensor with guanidinium hydrochloride for less than one minute. Guanidinium hydrochloride is known for its ability to break antibodyantigen complexes in immuno-affinity chromatography. While the sensor is not used in a measuring process, the cell ought to be filled with PBS-buffer (phosphate buffered saline), guaranteeing a longer life time of the antibody compared to storing it in air. This way the sensor gives results of the same quality for about 2 weeks; even if it is stored at room temperature (the life time is dependent on the temperature). After that time the antibody can be removed completely by washing with guanidinium hydrochloride for about half an hour. Finally, it is possible to apply a new coating by adding the antibody again. The new coating shows the same effect as the old one. Thus, calibration needs not to be done again.

\section{Measurement of sesame with antibody replica}
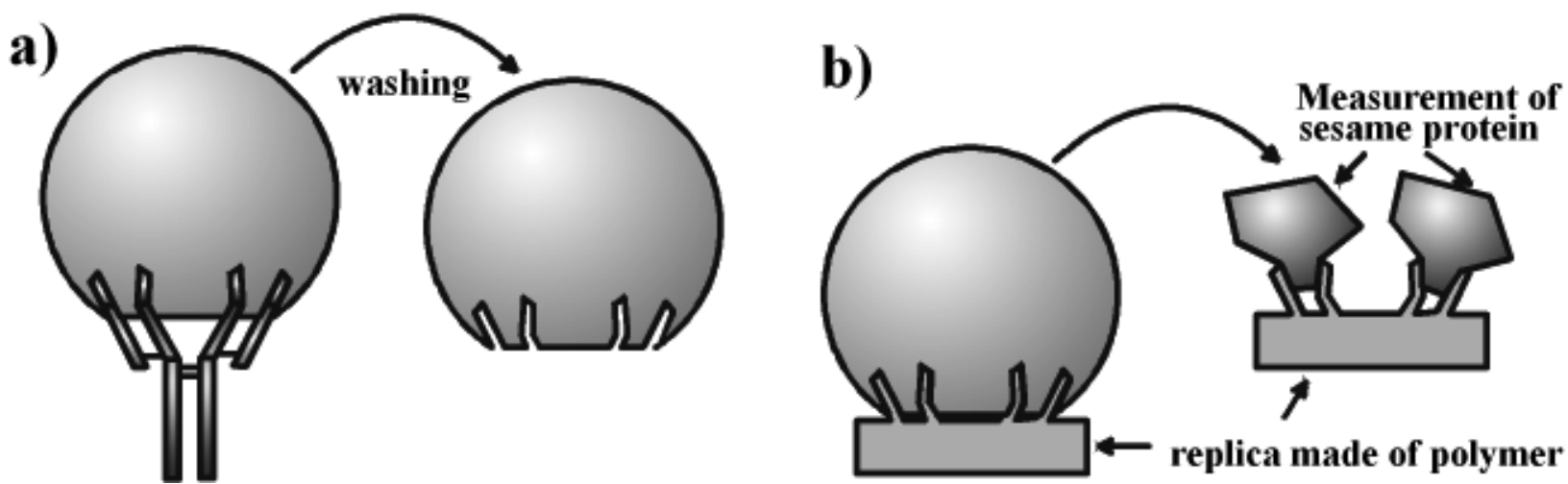

Fig. 2: a) preparation of printed nanoparticles b) their use for creation of an imprinted layer

Figure 2 shows the strategy which is to create imprinted nano particles. Thus, polymethacrylic acid polyvinylpyrollidone copolymer cross linked with dihydroxyethylenebisacylamide turned out to be advantageous. The cross linker hinders the shrinking and swelling in different solvents guaranteeing imprints with stable structure. Additionally, assembling more cross linker favours the formation of particles. After neutralisation with potassium hydroxide the pre-polymerisation can be performed in presence of the antibody. The neutralisation has to be done to prevent the protein from acidic denaturation. By replacing the natural antibody with an immunoglobulin targeting another antigen the procedure can be modified to detect other analytes.

After the pre-polymerisation nanoparticles were precipitated in acetonitrile. Nanobeads are favourably used since they show a large surface leading to high imprint density. ${ }^{10}$ Additionally these particles are easily removed from the surface. 
The most important task in molecular imprinting is to find a feasible polymer system. Therefore, the interaction sites of the polymer should be adjusted to the functional groups of the analyte. For the detection of pyrene for example polymers containing aromatic rings are suitable. ${ }^{11}$

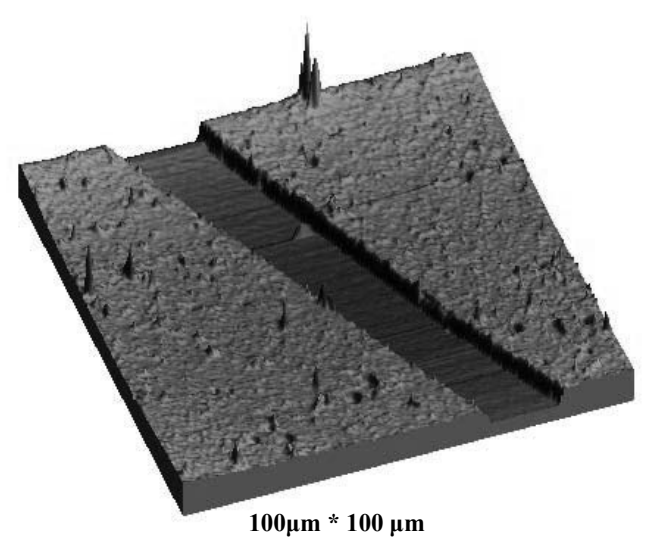

Fig. 3: Measurement of layer thickness with AFM
Layer thickness

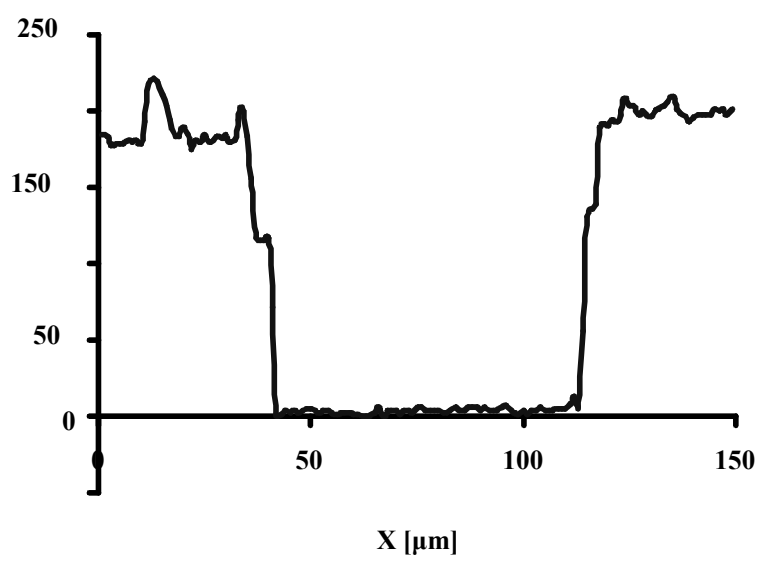

In the case of bioanalytes, which are known for their ability to form hydrogen bonds and hydrophobic binding sites, polymers capable to form hydrogen bonds have been tested. Further, it is important to use monomers which are soluble in water to prevent the pre-polymerisation in organic solvents leading

Sensor aus natürlichen Immunglobulinen mit Sesamprotein

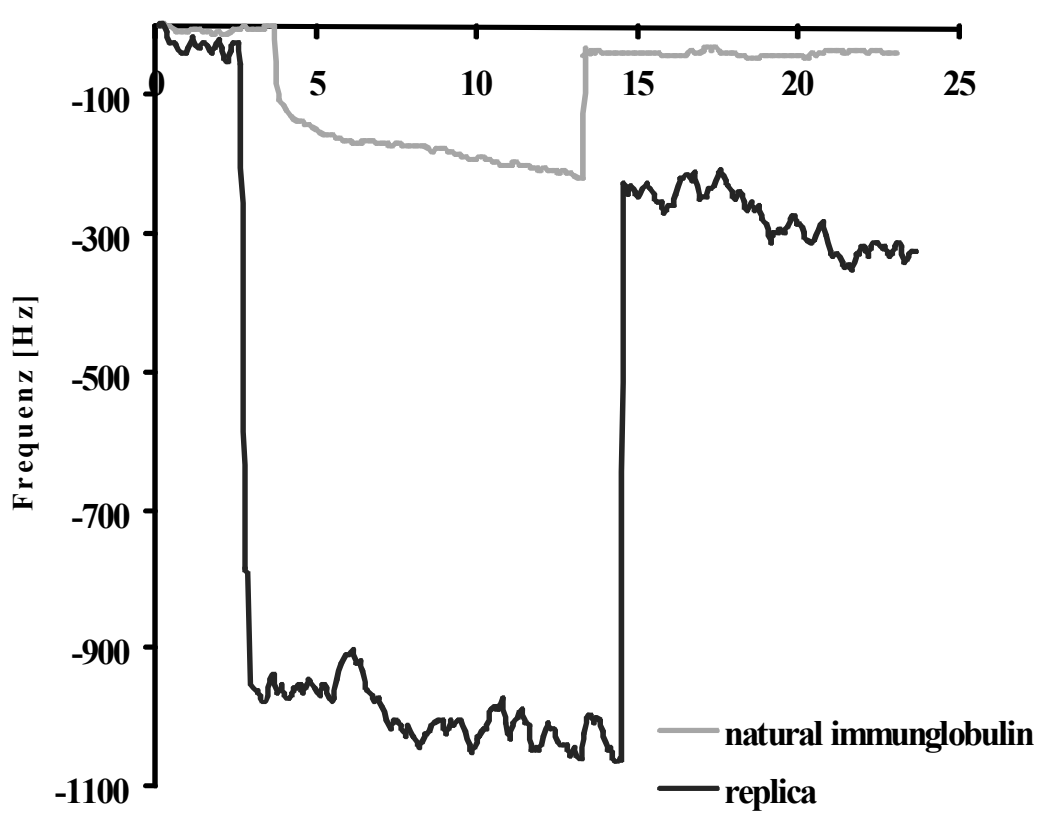

Zeit [min]

Fig. 4: Sensor responses for natural and artificial antibodies coated on a $10 \mathrm{MHz}$ QCM to denaturation of the antibody.

Additionally, the height of the deposited layer has to be adjusted to the particle size because layers of larger height lead to higher damping disturbing the measurement and hardens removing the stamp.

Reducing layer heights leads to lower surface roughness and thus minor sensor effects. The layer thickness can be adjusted by diluting the polymer with water. The height is tested by scratching the polymer and measuring with atomic force microscope (AFM). As it is shown in figure 3 the layer height is between 100 and $200 \mathrm{~nm}$.

As it is shown in figure 4 the replica gives a sensor response which is higher by a factor of approximately 10 and yields to an excellent selectivity, however, the same concentration of sesame $(0.11$ $\mathrm{mg} / \mathrm{ml}$ ) is added to both sensors. This result is very surprising since the QCM-answers observed are due to adsorption via an interfacial effect.

The use of nanoparticles is important since a high surface roughness is generated. Additionally, the formation of a poly layer is induced. 
Commercial food samples are complex and the analyte has to be detected under these conditions. To this end, the interferences of the matrix were studied in samples comprising sesame. Therefore, samples containing solid sedimenting particles or samples with high viscosities can cause

\section{Comparison of natural antibody and antibody replica}

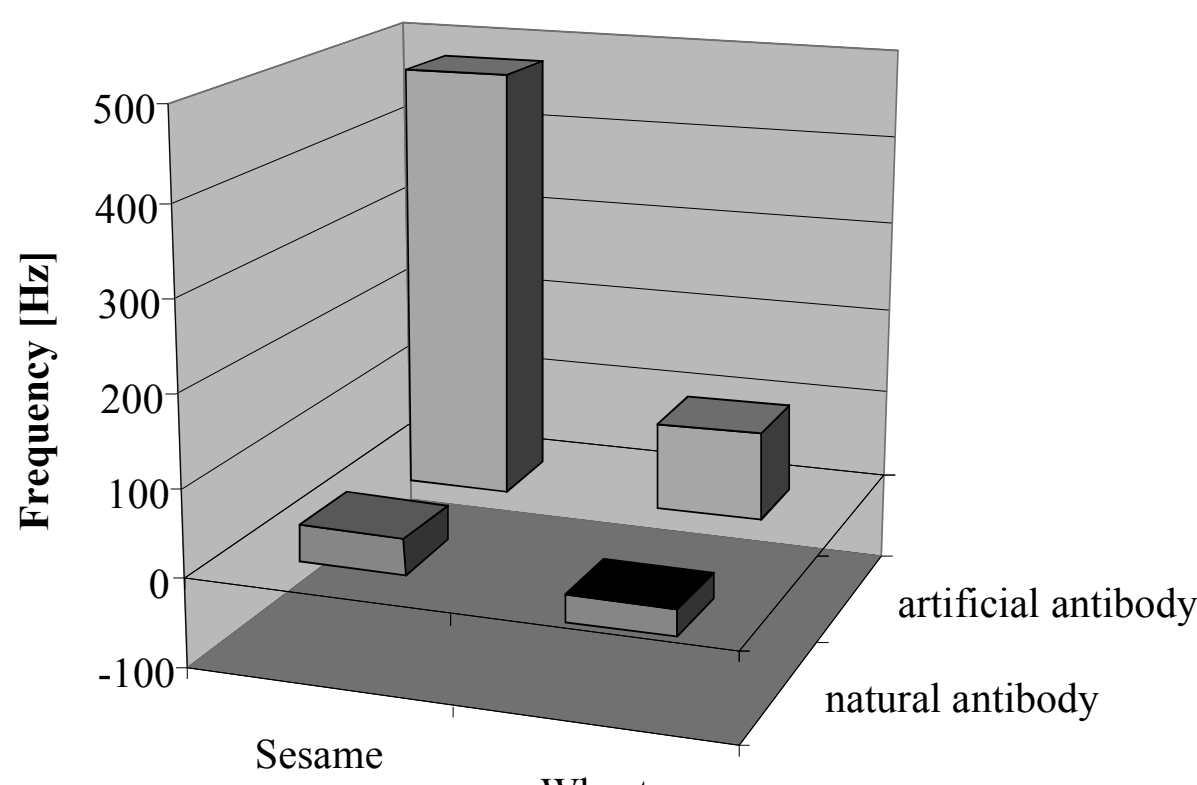

Wheat problems in the QCMmeasurement. Probes comprehending solid particles can be centrifuged to circumvent that problem. Samples with high viscosity or samples with extremely high ion concentration have to be diluted. Additionally, the effect of ions can be reduced by diminishing the electrode diameter of the counter electrode. The electrode geometry strongly influences ionic effects because it affects the course of the field lines. The effects are the less the more the electrodes differ in size. Finally, high selectivity can be achieved. Figure 5

Fig. 5: Responses of a $10 \mathrm{MHz}-\mathrm{QCM}$ coated with natural immunglobulin compared to antibody replica shows an example for the selectivity of the immunoglobulin replica compared to the natural antibody. It
can be seen that the response to wheat extract is low and good selectivity could be achieved. The replica gives an effect of approximately $500 \mathrm{~Hz}$, whereas the natural antibody gives just about $50 \mathrm{~Hz}$. Furthermore, the high cross selectivity of the plastic antibody to wheat, which gives only about $100 \mathrm{~Hz}$, is shown.

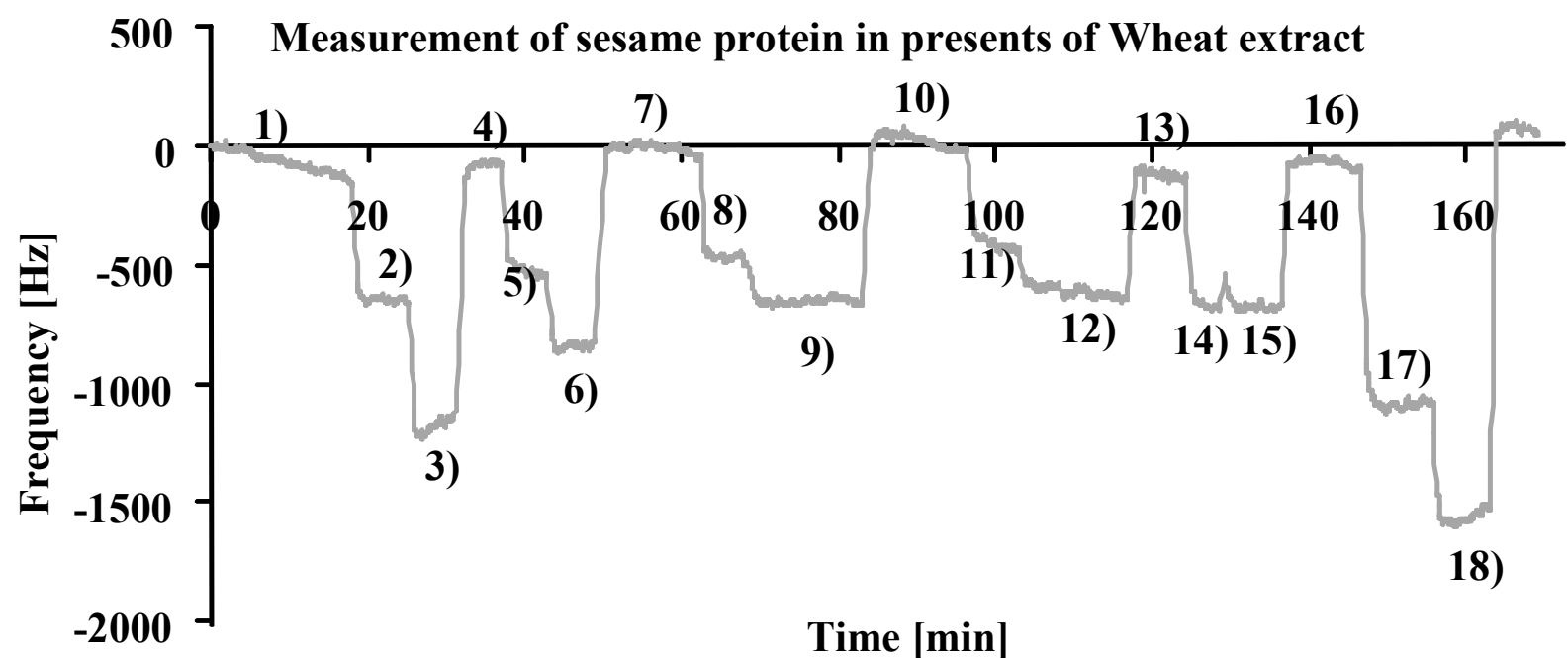

Fig. 6: Frequency response of a $10 \mathrm{MHz}$ QCM with antibody replica in extract of wheat. First water is injected (1) the decrease of the frequency is due to the incorporation of water into the layer. 2) shows the injection of wheat extract. Further, wheat spiked with sesame is injected and washed up with water again 3). 5) again shows the injection of wheat and 6) shows the injection of wheat spiked with sesame. The sensor is washed with water (7) again and wheat (8) and spiked wheat (9) are added and again washed up (10). 11) shows an other addition of wheat extract and 12) the injection of a spiked sample. After another washing step (13) wheat in higher concentration (14) is added and a spiked sample (15). 
The natural immunoglobulin shows a positive anti sauerbrey effect for wheat which is caused by non-bounded proteins on plane surfaces being able to "move around" on the surface. ${ }^{12,13}$

Additionally, the sensor responses shown in figure 6 give favourable reversibilities which were tested by washing with water after the measuring steps. Due to the fast binding the measurement lasts only a few minutes.

Furthermore, sesame was measured in complex matrices. Figure 6 shows an example for the measurement of sesame in presence of another analyte. It is depicted that wheat available in excess shows cross reactivity. To solve this problem it is necessary to have a suitable blank for the sample without any sesame. Therefore, food samples are injected as blank and spiked samples were tested. In figure 6 the the matrix was not altered, however, responses to different concentrations of sesame are observed.

\section{Conclusion:}

Artificial antibodies designed with the techniques of molecular imprinting have turned out to be a powerful tool to measure bio analytes. The sensitive layer can be produced without any complex synthesis steps by self assembling. Furthermore, the combination with quartz microbalance allows fast and label free detection. The resulting layers are more robust than their natural analogues which lead to a longer sensor lifetimes. Additionally, they show excellent selectivities. Surprisingly, the antibody replica shows even higher effects than the natural immunoglobulin immobilised on the gold electrodes of a QCM. Whereas the natural antibody shows an effect of $50 \mathrm{~Hz}$ the artificial one shows 10 times higher effect of $500 \mathrm{~Hz}$. This leads to a lower detection limit $(0.0002 \mathrm{mg} / \mathrm{ml})$ in comparison to the natural antibody $(0.007 \mathrm{mg} / \mathrm{ml})$. The reasons for such high effects are a high surface roughness and the formation of poly layers induced by the replica. In course of this procedure the particles are not consumed. Thus, only minor amounts of the expensive immunoglobulins as starting material are necessary for manufacturing a large number of sensors. Furthermore, these sensors can be used to detect sesame in complex food sample without knowledge of their exact composition. The procedure does not require any time consuming sample preparation steps. By replacing the natural antibody for the printing process of the beads with immunglobulins having selectivity to other antigens the process can be adjusted for various different biomolecules.

1 R. E. Poms, C. L. Klein, E. Anklam, Food Additives and Contaminants 1 (2004), 1-31

2 G. Kanny, C. De Hauteclocque, D.A. Moneret-Vautrin, Allergy 51, (1996)

3 K. Beyer, L. Bardina, G. Grishina, H. A. Sampson, Journal of Allergy and Clinical Immunology 110, (2002)

4 L.A. Johnson, T. M. Suleiman, E. W. Lusas, Journal of American oil chemists society 56 (1979), 463-468

5 F. L. Dickert, O. Hayden, R. Bindeus, K.-J. Mann, D. Blaas, E. Waigmann, Analytical and Bioanalytical Chemistry 378, (2004), 1929-1934

$6 \quad$ O. Hayden, R. Bindeus, C. Haderspöck, K. J. Mann, B. Wirl, F. L. Dickert, Sensors and Actuators B 91, (2003) 316-319

F. L. Dickert, P. Lieberzeit, S. Gazda-Miarecka, K. Halikias, K. J. Mann, Sensors and Actuators B 100 (2004) 112-116

8 O. Hayden, K.-J. Mann, S. Krassnig, F. L. Dickert, Angewandte Chemie International Edition 45 (2006)

9 P. A. Lieberzeit, A. Afzal, A. Rehman, F. L. Dickert, Sensors and Actuators B, (2007), 127-132

10 Z. Li, J. Ding, M. Day, Y. Tao, Macromolecules 39, (2006)

11 P. A. Lieberzeit, K. Halikias A. Afzal, F. L. Dickert, Analytical and Bioanalytical Chemistry 392 , (2008),1405-1410 1881

13 R. Lucklum, C. Behling, P. Hauptmann, Analytical Chemistry, Vol. 71, No. 13, (1999), 2488-2496 